Edinburgh/2002/01

\title{
Semi-leptonic decays of heavy mesons and the Isgur-Wise function in quenched lattice QCD
}

\author{
UKQCD Collaboration \\ K.C. Bowler ${ }^{a}$, G. Douglas ${ }^{a, 1}$, R.D. Kenway ${ }^{a}$, G.N. Lacagnina ${ }^{a}$, \\ C.M. Maynard ${ }^{\mathrm{a}}$ \\ ${ }^{a}$ Department of Physics $\&$ Astronomy, University of Edinburgh, Edinburgh EH9 \\ 3JZ, Scotland, UK
}

\begin{abstract}
The form factors for the semi-leptonic $B \rightarrow D$ and $B \rightarrow D^{*}$ decays are evaluated in quenched lattice QCD at two different values of the coupling, $\beta=6.0$ and 6.2. The action and the operators are fully $\mathcal{O}(a)$ non-perturbatively improved. The slope of the Isgur-Wise function is evaluated, and found to be $\rho^{2}=0.83_{-11-1}^{+15+24}$ (quoted errors are statistical and systematic respectively). Ratios of form factors are evaluated and compared to experimental determinations.
\end{abstract}

PACS numbers: 12.39.Hg, 13.20.He, 12.38.Gc, 11.10.Gh, 12.15.Hh

\section{Introduction}

The $B \rightarrow D$ and $B \rightarrow D^{*}$ semi-leptonic decays are of considerable phenomenological interest. They provide ample opportunity for interaction between experiment and theory. The ideas of Heavy Quark Symmetry (HQS) [1-5] and Heavy Quark Effective Theory (HQET) [6] were first developed and applied to these decays. A combination of theoretical and experimental data for semileptonic decays can be used to determine the CKM matrix element $\left|V_{c b}\right|$. HQS allows theoretical control of the non-perturbative (NP) aspects of the calculation around the infinite quark mass limit. In this study we determine the non-perturbative matrix elements directly on the lattice.

$\overline{1}$ CCIR, University of Edinburgh, 80 South Bridge, Edinburgh EH1 1HN, Scotland, UK 
HQS can be used to constrain the form of the matrix elements that describe semi-leptonic decays of heavy-light mesons. In particular, the relevant matrix elements are expressed in terms of a set of form factors that contain the nonperturbative physics of the decay:

$$
\begin{aligned}
\frac{\left\langle D\left(v^{\prime}\right)\left|\bar{c} \gamma^{\mu} b\right| B(v)\right\rangle}{\sqrt{m_{B} m_{D}}}= & \left(v+v^{\prime}\right)^{\mu} h_{+}(\omega)+\left(v-v^{\prime}\right)^{\mu} h_{-}(\omega) \\
\frac{\left\langle D^{*}\left(v^{\prime}, \epsilon\right)\left|\bar{c} \gamma^{\mu} b\right| B(v)\right\rangle}{\sqrt{m_{B} m_{D^{*}}}}= & i \epsilon^{\mu \nu \rho \sigma} \epsilon_{\nu}^{*} v_{\rho}^{\prime} v_{\sigma} h_{V}(\omega) \\
\frac{\left\langle D^{*}\left(v^{\prime}, \epsilon\right)\left|\bar{c} \gamma^{\mu} \gamma^{5} b\right| B(v)\right\rangle}{\sqrt{m_{B} m_{D^{*}}}}= & (\omega+1) \epsilon^{* \mu} h_{A_{1}}(\omega)+ \\
& -\left[h_{A_{2}}(\omega) v^{\mu}+h_{A_{3}}(\omega) v^{\prime \mu}\right]\left(\epsilon^{*} \cdot v\right)
\end{aligned}
$$

where $v, v^{\prime}$ are the velocities of the initial and the final meson respectively, and $\omega=v \cdot v^{\prime} ; \epsilon$ is the polarisation vector of the $D^{*}$ meson. The $\omega$ variable is kinematically constrained to the interval

$$
1 \leq \omega \leq \frac{m_{B}^{2}+m_{D^{(*)}}^{2}}{2 m_{B} m_{D^{(*)}}} .
$$

In the limit of infinitely heavy bottom and charm quarks, the six form factors are related to a universal function known as the Isgur-Wise function $\xi(\omega)[4,5]$. Away from the heavy quark limit this relation is modified by two kinds of corrections: perturbative QCD corrections and heavy quark symmetry breaking corrections. For large enough heavy quark masses, the relationships between the form factors and $\xi(\omega)$ are

$$
h_{j}(\omega)=\left[\alpha_{j}+\beta_{j}\left(m_{b}, m_{c} ; \omega\right)+\gamma_{j}\left(m_{b}, m_{c} ; \omega\right)+\mathcal{O}\left(1 / m_{b, c}^{2}\right)\right] \xi(\omega)
$$

The $\alpha_{j}$ terms are constants that fix the behaviour of the form factors in the heavy quark limit $\left(\alpha_{+}=\alpha_{V}=\alpha_{A_{1}}=\alpha_{A_{3}}=1 ; \alpha_{-}=\alpha_{A_{2}}=0\right)$. The $\beta_{j}$ and $\gamma_{j}$ functions account respectively for radiative corrections and power corrections proportional to the inverse of the heavy quark mass. The radiative corrections are calculable in perturbation theory [7], while the power corrections are nonperturbative in nature [8]. At zero recoil $\left(v=v^{\prime}, \omega=1\right)$ however, Luke's theorem [9] guarantees that $\gamma_{+}=0$ and $\gamma_{A_{1}}=0$, which means that power corrections to $h_{+}$and $h_{A_{1}}$ are of order $\mathcal{O}\left(1 / m_{b, c}^{2}\right)$.

At small recoil, the Isgur-Wise function is modelled by

$$
\xi(\omega)=1-\rho^{2}(\omega-1)+\mathcal{O}\left((\omega-1)^{2}\right)
$$


where $\rho^{2}$ is called the slope parameter and $\xi(1)=1$, because of current conservation. Alternative parametrisations of $\xi(\omega)$ are possible, which start to differ from $(6)$ at $\mathcal{O}\left((\omega-1)^{2}\right)[10,11]$.

In this paper we present a study of the form factors of the $B \rightarrow D$ and $B \rightarrow D^{*}$ semi-leptonic decays, and the extraction of the Isgur-Wise function in the quenched approximation to lattice QCD. The calculations are performed at two values of the coupling, $\beta=6.0$ and $\beta=6.2$, using a non-perturbatively improved relativistic Sheikholeslami-Wohlert (SW) [12] fermion action and current operators, so that the leading discretisation errors appear at $\mathcal{O}\left(a^{2}\right)$ rather than $\mathcal{O}(a)$, where $a$ is the lattice spacing. Whilst the improvement gives better control over the continuum extrapolation, it does not necessarily reduce the size of lattice artifacts at fixed coupling. With only two values of the coupling, no continuum extrapolation is attempted.

The remainder of the paper is organised as follows. In section 2 we give details of the calculation including extraction of the form factors. In section 3 we describe our determination of the vector and axial current renormalisation constants. In section 4 we report the results of the extraction of the IsgurWise function and its slope. Section 5 dwells on the problem of quark mass dependence, while section 6 describes a calculation of ratios of form factors and its comparison with experimental determinations. An analysis of the systematic sources of uncertainty and a comparison with other results is detailed in section 7 .

\section{Details of the calculation}

\subsection{Improvement of action, currents and masses}

The improved action used in this work has the form

$$
S_{\mathrm{SW}}=S_{\mathrm{W}}-c_{\mathrm{SW}} \frac{i \kappa}{2} \sum_{x} \bar{\psi}(x) i \sigma_{\mu \nu} F_{\mu \nu}(x) \psi(x)
$$

where $S_{\mathrm{W}}$ is the Wilson action. The improvement programme also requires the improvement of current operators; in particular, the improved vector and axial currents are

$$
\begin{aligned}
& V_{\mu}^{\mathrm{I}}(x)=V_{\mu}(x)+a c_{V} \tilde{\partial}_{\nu} T_{\mu \nu}(x) \\
& A_{\mu}^{\mathrm{I}}(x)=A_{\mu}(x)+a c_{A} \tilde{\partial}_{\mu} P(x)
\end{aligned}
$$


where

$$
\begin{aligned}
V_{\mu}(x) & =\bar{\psi}(x) \gamma_{\mu} \psi(x) \\
A_{\mu}(x) & =\bar{\psi}(x) \gamma_{\mu} \gamma_{5} \psi(x) \\
P(x) & =\bar{\psi}(x) \gamma_{5} \psi(x) \\
T_{\mu \nu}(x) & =\bar{\psi}(x) i \sigma_{\mu \nu} \psi(x)
\end{aligned}
$$

and $\tilde{\partial}_{\mu}$ is the symmetric lattice derivative in the $\hat{\mu}$ direction, defined in terms of the lattice spacing $a$ by

$$
\tilde{\partial}_{\mu} f(x)=\frac{1}{2 a}[f(x+a \hat{\mu})-f(x-a \hat{\mu})] .
$$

The generic renormalisation of improved current operators is performed as follows $(J=A, V)$ :

$$
J^{\mathrm{R}}=Z_{J}\left(g^{2}\right)\left(1+b_{J}\left(g^{2}\right) a m_{q}\right) J^{\mathrm{I}}
$$

where $Z_{J}$ is calculated in a mass-independent renormalisation scheme. It is conventional to define effective renormalisation constants:

$$
Z_{J}^{\mathrm{eff}}=Z_{J}\left(1+b_{J} a m_{q}\right)
$$

The bare quark mass, in terms of hopping parameters, is equal to

$$
a m_{q}=\frac{1}{2}\left(\frac{1}{\kappa_{q}}-\frac{1}{\kappa_{\text {crit }}}\right)
$$

where $\kappa_{\text {crit }}$ is the value of the hopping parameter at which the bare quark mass vanishes. The improved, renormalisation group invariant (RGI) quark mass is defined in the following way

$$
a \tilde{m}_{q}=Z_{m} a m_{q}\left(1+b_{m} a m_{q}\right) .
$$

A discussion of the different determinations of the improvement coefficients can be found in [13]. We use the NP value of $c_{\mathrm{SW}}$ determined by the ALPHA collaboration $[14,15]$ and the improvement coefficients for the current operators are taken from Bhattacharya et al. [16-18], except for $Z_{m}$ and $b_{m}$. The additive $b_{m}$ coefficient is evaluated at 1-loop in perturbation theory [19] with the coupling "boosted" by the mean link, $g^{2} \rightarrow g^{2} / u_{0}^{4}$, and $Z_{m}$ is determined non-perturbatively by the ALPHA collaboration [20]. 
Table 1

Simulation details.

\begin{tabular}{lcc}
\hline & $\beta=6.2$ & $\beta=6.0$ \\
\hline Volume & $24^{4} \times 48$ & $16^{3} \times 48$ \\
$N_{\text {configs }}$ & 216 & 305 \\
$c_{\mathrm{S} W}$ & 1.614 & 1.769 \\
$a^{-1}\left(r_{0}\right)(\mathrm{MeV})$ & 2.913 & 2.123 \\
$\kappa_{\text {heavy }}$ & $0.120,0.1233,0.1266,0.1299$ & $0.1123,0.1173,0.1223,0.1273$ \\
$\kappa_{\text {light }}$ & $0.1346,0.1351$ & $0.13344,0.13417$ \\
& & \\
\hline
\end{tabular}

\subsection{Simulation details}

The lattice spacing is set using the Sommer scale $r_{0}[21,22]$. The matrix elements are extracted from combinations of quark propagators corresponding to four values of the heavy quark masses $\left(m_{\text {heavy }} \simeq m_{\mathrm{c}}\right)$ and two values of the mass of the light (passive) quark $\left(m_{\text {light }} \simeq m_{\mathrm{s}}\right)$. Simulation details are summarised in Table 1. The gauge configurations that are used in this calculation were generated with a combination of the over-relaxed [23,24] and the Cabibbo-Marinari [25] algorithms with periodic boundary conditions. The light quark propagators are smeared with the fuzzing technique [26], while heavy quark propagators are smeared using a gauge invariant technique [27]. The statistical errors are estimated using the bootstrap method [28].

\subsection{Extraction of the form factors}

The matrix elements relevant to the semi-leptonic decays are extracted from fits to two and three-point correlation functions. The general form of the latter is as follows

$$
C_{3 \mathrm{pt}, J}^{\mu}\left(\vec{p}_{A}, t_{x} ; \vec{p}_{B}, t_{y}\right)=\sum_{\vec{x}, \vec{y}} e^{-i\left(\vec{p}_{B} \cdot \vec{x}+\vec{q} \cdot \vec{y}\right)}\left\langle 0\left|T\left[\Omega_{B}(x) J^{\mu \dagger}(y) \Omega_{A}^{\dagger}(0)\right]\right| 0\right\rangle
$$

where $\vec{q}=\vec{p}_{B}-\vec{p}_{A}$ and $\Omega_{X}^{\dagger}$ is the operator that creates the state $X . J^{\mu}$ is the weak current. We have computed the correlation function using the "standard source" method [29,30]. The time-slice $t_{x}$ is fixed to $t_{x}=28$, i.e. slightly off the centre of the lattice, in order to allow for an analysis of the different systematic effects of the two sides of the lattice. The extended heavy quark propagators were computed at four values of the hopping parameter. For the active quark 
propagators, which are part of the current, only two values of the hopping parameter were used at $\beta=6.2$ due to disk space constraints and all four were used at $\beta=6.0$.

In the Euclidean time formulation, when the time separation of the operators is large enough, i.e. when $t_{y}$ and $t_{x}-t_{y}$ are large, one has

$$
C_{3 \mathrm{pt}, J}^{\mu}\left(\vec{p}_{A}, t_{x} ; \vec{p}_{B}, t_{y}\right)=\frac{Z_{A}}{2 E_{A}} e^{-E_{A} t_{y}} \frac{Z_{B}}{2 E_{B}} e^{-E_{B}\left(t_{x}-t_{y}\right)}\left\langle B\left(\vec{p}_{B}\right)\left|J^{\mu \dagger}(0)\right| A\left(\vec{p}_{A}\right)\right\rangle(15
$$

where $Z_{i}=\left\langle 0\left|P_{i}\right| P_{i}\left(\vec{p}_{i}\right)\right\rangle$. The $Z_{i}$ constants and the energies $E_{i}=\sqrt{\left|\vec{p}_{i}\right|^{2}+m_{i}^{2}}$ are extracted from fits of two-point functions (detailed descriptions of the methods employed can be found in $[13,31])$. The $\left\{Z_{i}, E_{i}\right\}$ parameters are then used to cancel the asymptotic time dependence from the three-point function and to extract the desired matrix element from a fit to a plateau. For each value of $\omega$ where

$$
\omega=\frac{m_{B}^{2}+m_{D^{(*)}}^{2}-q^{2}}{2 m_{B} m_{D^{(*)}}}
$$

an average is performed of all the kinematic and Lorentz channels for which the matrix elements have the same value. An example matrix element is shown in figure 1. All distinct matrix elements are fitted simultaneously to extract the form factors. We examine five kinematic channels, each of them being specified by the values of $\left|\vec{p}_{A}\right|,|\vec{q}|$. These are summarised in Table 2 .

Since the heavy quarks used in this study have masses around that of the charm quark, we are in effect extracting the Isgur-Wise function from a $D \rightarrow$ $D^{(\star)}$ decay. Provided that there is little residual mass dependence beyond leading order power and radiative corrections in the form factors, then the machinery of HQS allows us to compare to experimental $B \rightarrow D^{(\star)}$ decays.

Table 2

Spatial moduli of momenta in the different kinematic channels, in lattice units of $2 \pi /(a L)$.

\begin{tabular}{ccc}
\hline Channel \# & $\left|\vec{p}_{A}\right|$ & $|\vec{q}|$ \\
\hline 0 & 0 & 0 \\
1 & 0 & 1 \\
2 & 1 & 1 \\
3 & 1 & 0 \\
4 & 1 & $\sqrt{2}$ \\
\hline
\end{tabular}



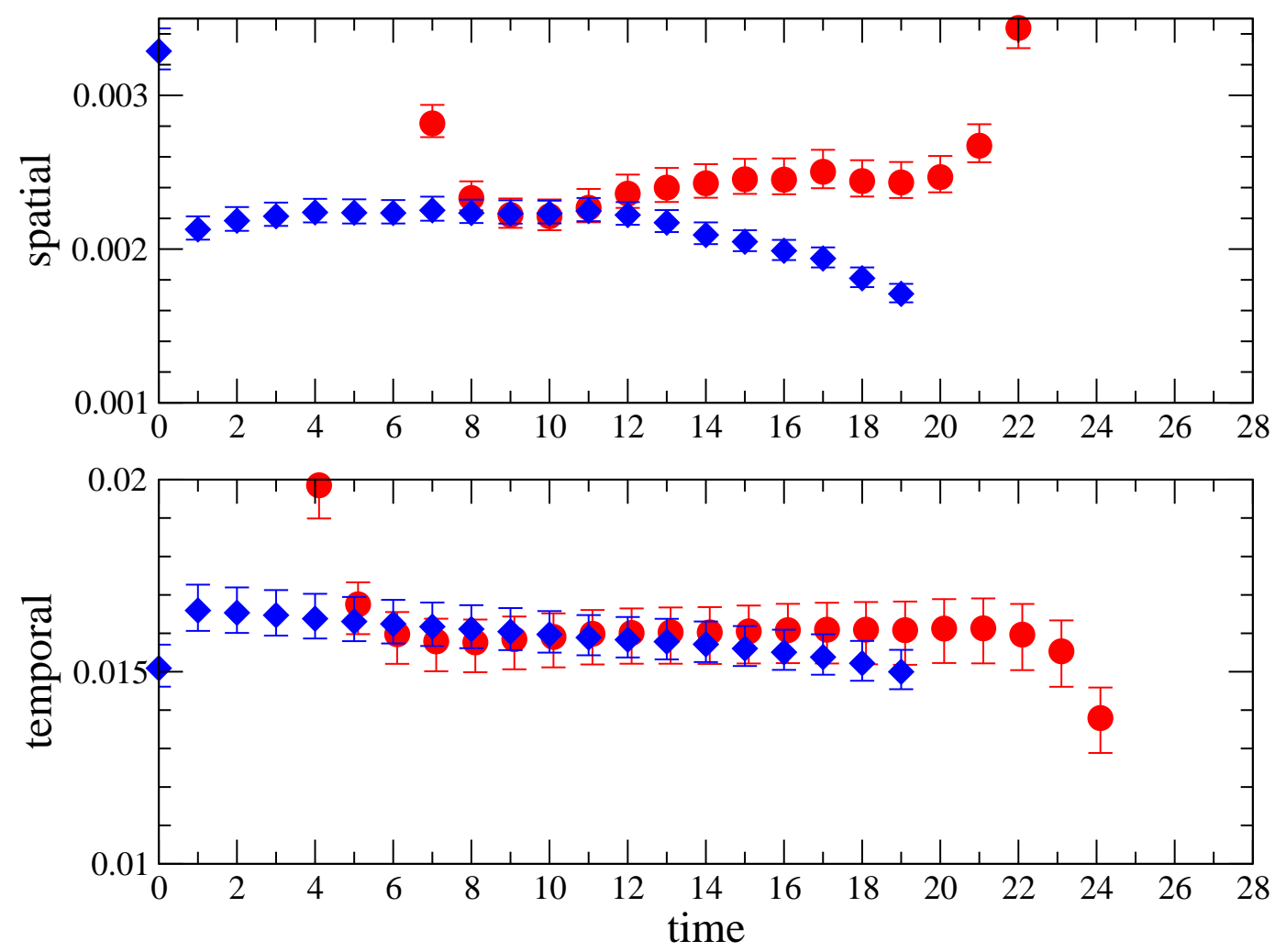

Fig. 1. The matrix element $\left\langle P_{B}\left(\vec{p}_{B}\right)\left|V_{\mu}\right| P_{A}\left(\vec{p}_{A}\right)\right\rangle$ with $\beta=6.2, \kappa_{H}=\kappa_{H^{\prime}}=0.1200$, $\kappa_{L}=0.1346,\left|\vec{p}_{A}\right|=0$ and $\left|\vec{p}_{B}\right|=1$. The data shows the ratio of the three-point function over the fitted two-point parameters. The circles are $0<t_{y}<27$ (fore side) and the diamonds are $0<\left(T-t_{y}\right)<19$ (back side).

\section{Current renormalisation}

\subsection{The vector current}

Observing that the degenerate mass vector current is conserved in the continuum, we consider the effective matching coefficient $Z_{V}^{\text {eff }}$, obtained from the forward matrix element of the current between degenerate heavy-light mesons at rest. We then have

$$
\left\langle P(\overrightarrow{0})\left|V_{0}\right| P(\overrightarrow{0})\right\rangle^{\text {cont }}=2 M=Z_{V}^{\text {eff }}\left\langle P(\overrightarrow{0})\left|V_{0}^{I}\right| P(\overrightarrow{0})\right\rangle^{\text {latt }} .
$$

In particular, taking the ratio of a heavy-light two-point function, at the extension time-slice, with the forward degenerate matrix element gives,

$$
\frac{C_{2 \mathrm{pt}}\left(\vec{p}=\overrightarrow{0} ; t=t_{x}\right)}{C_{3 \mathrm{pt}}^{A=B}\left(\overrightarrow{p_{A}}=\overrightarrow{p_{B}}=\overrightarrow{0} ; t_{x}, t_{y}\right)}=\frac{Z^{2} e^{-M t_{x}}}{2 M} \times
$$


Table 3

$Z_{V}^{\text {eff }}$ and quark mass. $a m_{Q}$ is the bare quark mass appearing in the current and $a m_{q}$ is the bare quark mass of the light, spectator quark.

\begin{tabular}{|c|c|c|c|}
\hline$\beta$ & $a m_{Q}$ & \multicolumn{2}{|c|}{$Z_{V}^{\mathrm{eff}}$} \\
\hline \multirow{3}{*}{6.2} & & $a m_{q}=0.0332$ & $a m_{q}=0.0195$ \\
\hline & 0.485 & $1.3122_{-3}^{+3}$ & $1.3111_{-3}^{+3}$ \\
\hline & 0.268 & $1.0871_{-3}^{+3}$ & $1.0873_{-4}^{+4}$ \\
\hline \multirow{5}{*}{6.0} & & $a m_{q}=0.0502$ & $a m_{q}=0.0298$ \\
\hline & 0.756 & $1.5963_{-8}^{+8}$ & $1.5946_{-13}^{+11}$ \\
\hline & 0.566 & $1.3876_{-6}^{+6}$ & $1.3843_{-10}^{+9}$ \\
\hline & 0.392 & $1.2058_{-4}^{+3}$ & $1.2044_{-5}^{+5}$ \\
\hline & 0.231 & $1.0333_{-3}^{+3}$ & $1.0333_{-3}^{+3}$ \\
\hline \multicolumn{4}{|r|}{$(2 M)^{2}$} \\
\hline & & \multicolumn{2}{|c|}{ 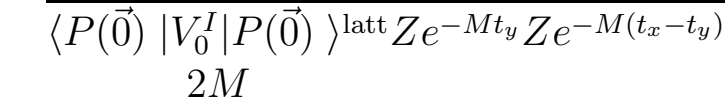 } \\
\hline & & \multicolumn{2}{|c|}{$\begin{array}{l}=\overline{\left\langle P(\overrightarrow{0})\left|V_{0}^{I}\right| P(\overrightarrow{0})\right\rangle^{\text {latt }}} \\
=Z^{\mathrm{eff}}\end{array}$} \\
\hline
\end{tabular}

The results as a function of bare quark mass, as it appears in equation (11), are given in Table 3. Also shown in the table is the spectator quark mass. We find that the current renormalisation does not depend significantly on the spectator quark mass. The results for $\beta=6.2$ have already been reported in [32]. We note a small discrepancy that is accounted for by the following: in [32] we used the fitted two-point function values of the mass and overlap factor, whereas here we use the two-point function itself to determine $Z_{V}^{\text {eff }}$. This is expected to reduce the statistical errors as the ratio is independent of $t_{y}$.

\subsection{The axial current}

The axial current is not conserved, and so we rely on HQS to determine the effective renormalisation constant. The form factor $h_{A_{1}}$ at zero recoil is equal to unity, up to symmetry breaking corrections, which are reduced to $\mathcal{O}\left(1 / m_{Q}^{2}\right)$ by Luke's theorem. This form factor can be measured on the lattice, and is related to the effective renormalisation by,

$$
Z_{A}^{\mathrm{eff}} h_{A_{1}}^{\mathrm{latt}}(1)=1+\beta_{A_{1}}(1)+\mathcal{O}\left(1 / m_{Q}^{2}\right)
$$



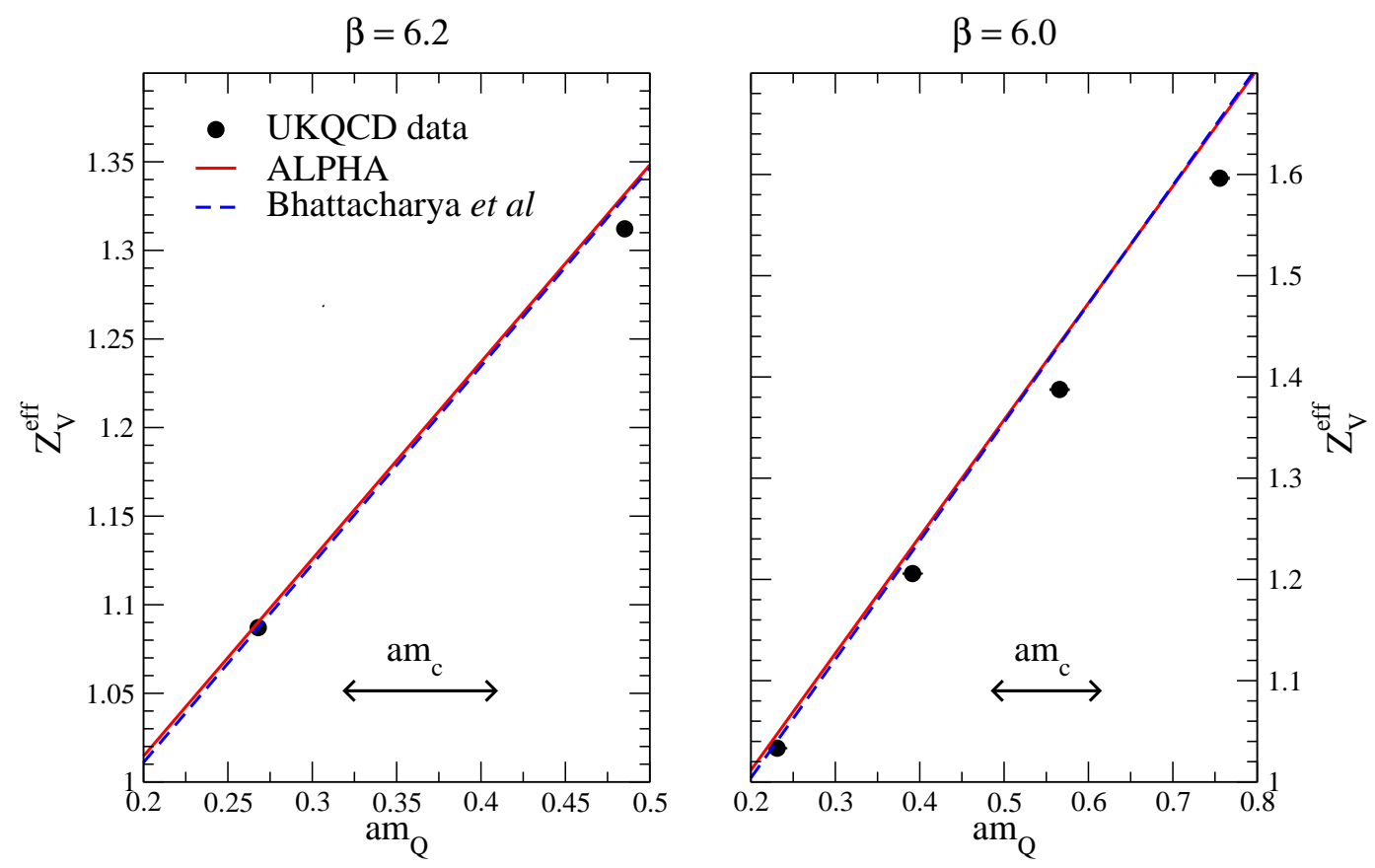

Fig. 2. $Z_{V}^{\text {eff }}$ versus bare quark mass at both values of $\beta . a m_{c}$ is the charm quark mass in lattice units, as extracted from a lattice determination of the heavy-light pseudoscalar meson mass. The range indicates the variation in the mass due to the different choices of the scale-fixing quantity.

To measure the form factor, we do not rely on degenerate initial and final states only. When they are not degenerate, the renormalisation depends on the average mass of the quarks in the current. Our results are shown in Table 4. Entries that have the repeated average quark mass are taken from matrix elements with the initial and final states interchanged.

\subsection{Comparison with other determinations}

We compare our measurement of the effective renormalisation to equation (11) using values of $Z_{J}$ and $b_{J}$ determined non-perturbatively by the ALPHA collaboration [33] and Bhattacharya et al. [16-18]. This comparison for the vector current is shown in Figure 2 for the heaviest spectator quark. The agreement is quite striking, especially considering that the ALPHA collaboration determined the coefficients at near zero quark mass and Bhattacharya et al for light quarks (strange scale). Indeed, Bhattacharya et al. have already compared their results at $\beta=6.2$ to those in [32].

For the axial current, there is no NP determination of $b_{A}$ by the ALPHA collaboration. To facilitate a comparison, we use the one-loop value of $b_{A}[19]$

$$
b_{A}=1+0.1522 g^{2}
$$


Table 4

$Z_{A}^{\text {eff }}$ and average heavy quark mass.

\begin{tabular}{|c|c|c|c|}
\hline$\beta$ & $\left\langle a m_{Q}\right\rangle$ & & \\
\hline & & $a m_{q}=0.0332$ & $a m_{q}=0.0195$ \\
\hline \multirow{8}{*}{6.2} & 0.485 & $1.29_{-3}^{+3}$ & $1.27_{-4}^{+4}$ \\
\hline & 0.429 & $1.26_{-3}^{+3}$ & $1.25_{-3}^{+4}$ \\
\hline & 0.377 & $1.24_{-3}^{+3}$ & $1.22_{-3}^{+4}$ \\
\hline & 0.377 & $1.23_{-3}^{+3}$ & $1.23_{-3}^{+4}$ \\
\hline & 0.326 & $1.20_{-3}^{+3}$ & $1.19_{-3}^{+3}$ \\
\hline & 0.321 & $1.18_{-3}^{+3}$ & $1.17_{-3}^{+4}$ \\
\hline & 0.268 & $1.10_{-2}^{+3}$ & $1.09_{-3}^{+3}$ \\
\hline & \multirow[t]{2}{*}{0.218} & $1.10_{-3}^{+2}$ & $1.09_{-3}^{+3}$ \\
\hline \multirow{17}{*}{6.0} & & $a m_{q}=0.0502$ & $a m_{q}=0.0298$ \\
\hline & 0.756 & $1.55_{-5}^{+5}$ & $1.52_{-7}^{+7}$ \\
\hline & 0.661 & $1.45_{-5}^{+5}$ & $1.43_{-6}^{+6}$ \\
\hline & 0.661 & $1.48_{-5}^{+5}$ & $1.46_{-7}^{+7}$ \\
\hline & 0.573 & $1.40_{-4}^{+5}$ & $1.38_{-6}^{+5}$ \\
\hline & 0.573 & $1.43_{-5}^{+5}$ & $1.42_{-7}^{+7}$ \\
\hline & 0.566 & $1.37_{-5}^{+4}$ & $1.36_{-6}^{+5}$ \\
\hline & 0.493 & $1.33_{-4}^{+4}$ & $1.31_{-5}^{+5}$ \\
\hline & 0.493 & $1.41_{-4}^{+5}$ & $1.41_{-7}^{+7}$ \\
\hline & 0.479 & $1.32_{-4}^{+4}$ & $1.31_{-5}^{+5}$ \\
\hline & 0.479 & $1.33_{-4}^{+4}$ & $1.32_{-6}^{+6}$ \\
\hline & 0.398 & $1.27_{-4}^{+4}$ & $1.25_{-5}^{+4}$ \\
\hline & 0.398 & $1.20_{-4}^{+4}$ & $1.32_{-6}^{+6}$ \\
\hline & 0.391 & $1.22_{-4}^{+4}$ & $1.21_{-5}^{+5}$ \\
\hline & 0.311 & $1.19_{-4}^{+4}$ & $1.18_{-4}^{+4}$ \\
\hline & 0.311 & $1.23_{-4}^{+4}$ & $1.23_{-5}^{+5}$ \\
\hline & 0.231 & $1.03_{-3}^{+3}$ & $1.01_{-4}^{+4}$ \\
\hline
\end{tabular}

with the "boosted" coupling, $g^{2}=g_{0}^{2} / u_{0}^{4}$, where the mean link, $u_{0}$, is evaluated from the 4 th root of the average plaquette. This is shown in Figure 3. We also show the values of $Z_{A}^{\text {eff }}$ evaluated in boosted perturbation theory (BPT). Again, our data compares very well to other determinations. 

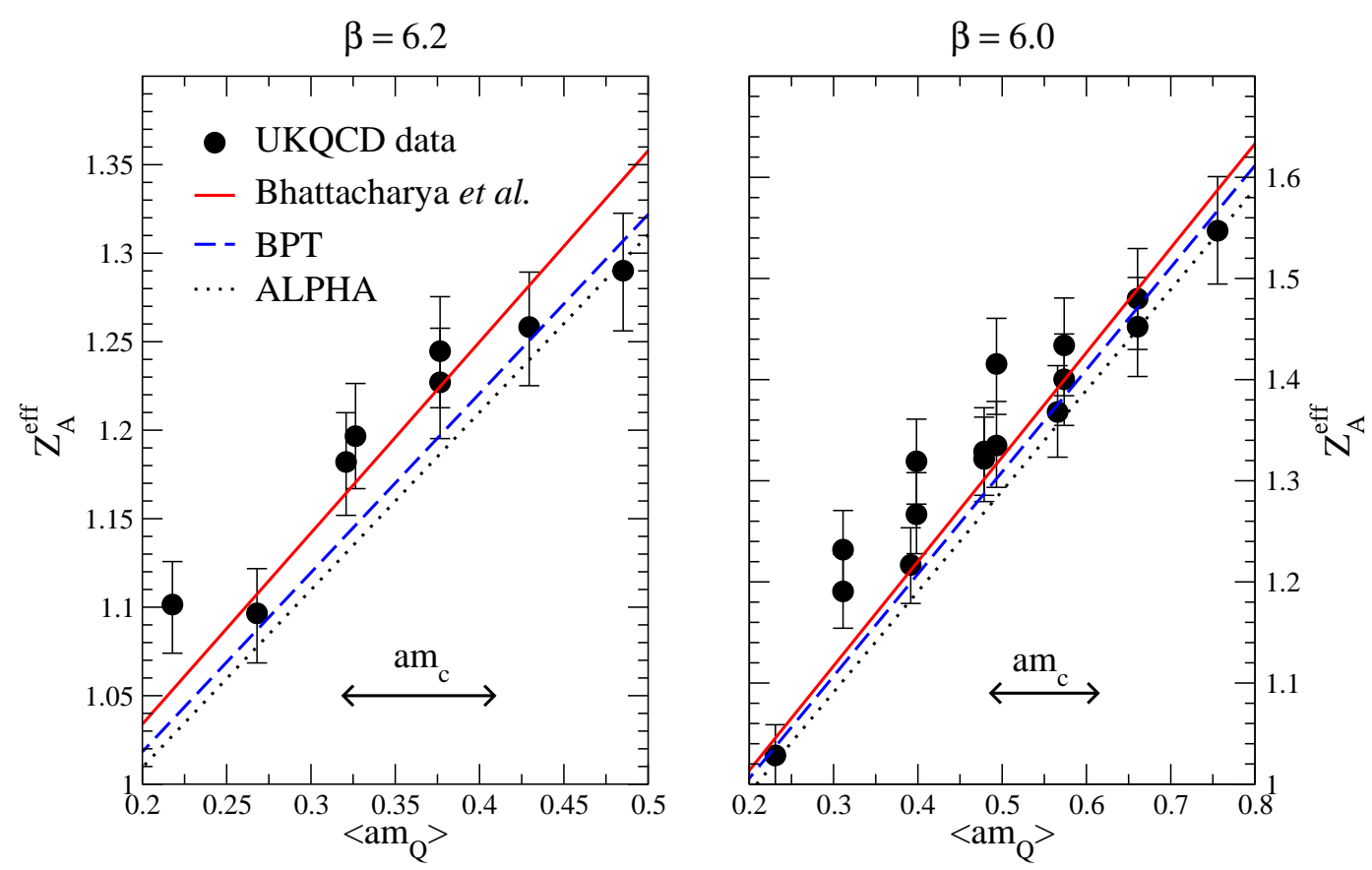

Fig. 3. $Z_{A}^{\text {eff }}$ against average bare quark mass.

We don't use our results to determine $b_{J}$ and $Z_{J}$; however, the comparison suggests that lattice artifacts are not spoiling the renormalisation.

\section{Extraction of the Isgur-Wise function}

The form factors that are protected from power corrections by Luke's theorem are also used to extract the Isgur-Wise function:

$$
\begin{aligned}
& \xi(\omega) \simeq \frac{h_{+}(\omega)}{1+\beta_{+}(\omega)}, \\
& \xi(\omega) \simeq \frac{h_{A_{1}}(\omega)}{1+\beta_{A_{1}}(\omega)} .
\end{aligned}
$$

The power corrections for $h_{+}$and $h_{A_{1}}$ have been estimated and found to be consistent with zero in the range $1.0 \leq \omega \leq 1.2$, and therefore are neglected. It is not possible to extract the Isgur-Wise function from the form factors for which $\alpha_{j}=0$ as they differ from zero only by power corrections. In principle it would be possible to measure the power corrections in this way. However, the poor quality of the data for the sub-leading form factors prevents this. Similarly, the $h_{V}$ form factor could not be used because its power correction is found to be large, as expected [7]. 


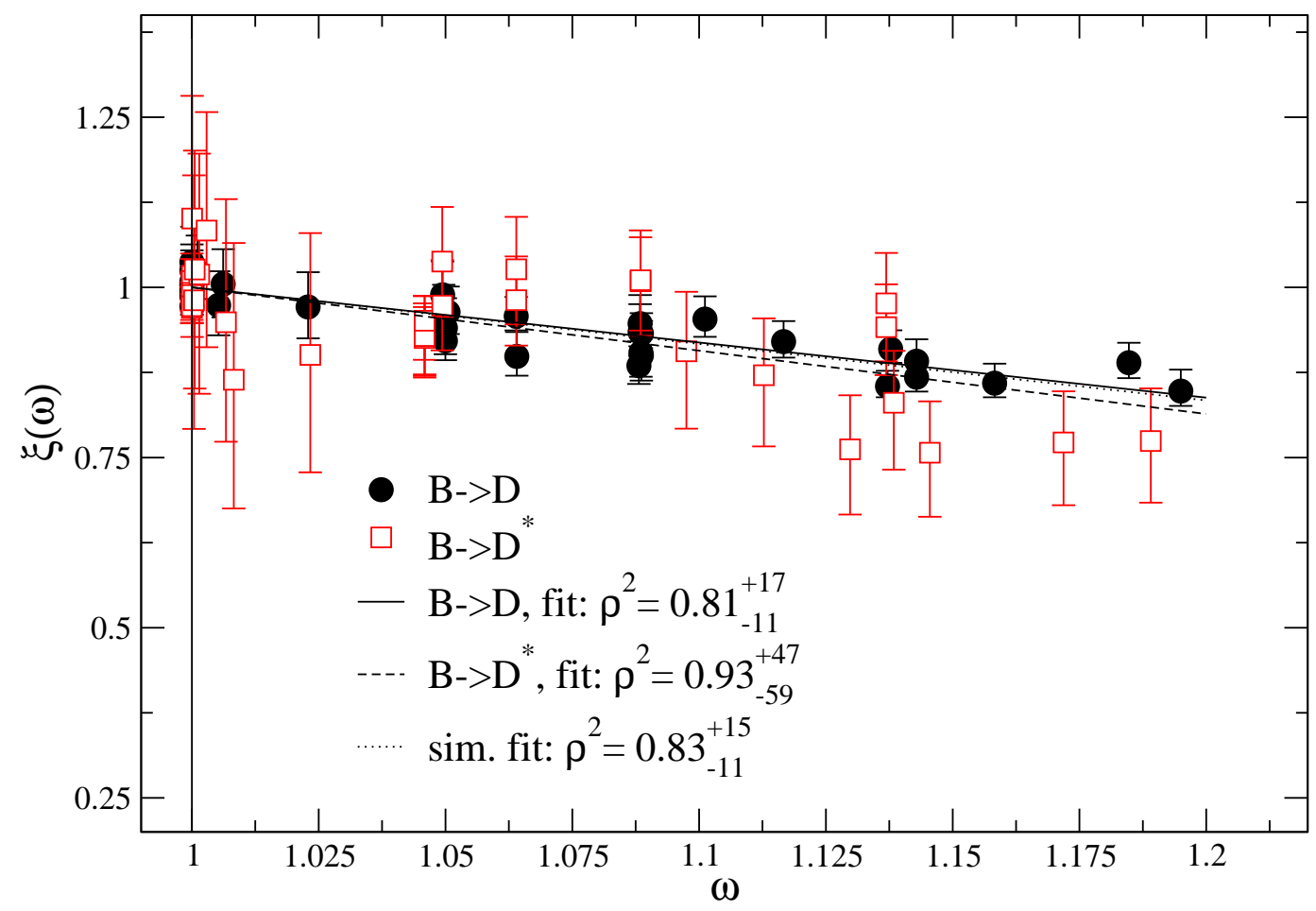

Fig. 4. The Isgur-Wise function from the $B \rightarrow D$ and $B \rightarrow D^{*}$ semi-leptonic decays, with $\kappa_{\text {light }}=0.1351$, including single-decay and simultaneous fits.

Table 5

Results of the linear fits to the Isgur-Wise function, at $\beta=6.2$. Quoted errors are statistical.

\begin{tabular}{lcccccc}
\hline & \multicolumn{2}{c}{$B \rightarrow D$} & \multicolumn{2}{c}{$B \rightarrow D^{*}$} & \multicolumn{2}{c}{ Sim. fit } \\
$\kappa_{\text {light }}$ & 0.1346 & 0.1351 & 0.1346 & 0.1351 & 0.1346 & 0.1351 \\
\hline$\rho^{2}$ & $0.97_{-9}^{+14}$ & $0.81_{-11}^{+17}$ & $1.43_{-32}^{+15}$ & $0.93_{-59}^{+47}$ & $1.06_{-11}^{+11}$ & $0.83_{-11}^{+15}$ \\
\hline
\end{tabular}

The Isgur-Wise function is fitted to the linear model (6), at both values of the coupling. Simultaneous fits of the Isgur-Wise function from the two distinct decays are also performed; the assumption is made that the effect of mixing different systematic errors (different decays) is no larger than the effect that one gets by fitting simultaneously data from correlators with different momenta. Results are summarised in Tables 5,6 and plotted in Figure 4. The determination of the slope of the Isgur-Wise function at $\beta=6.2$ from the simultaneous fit of the two form factors, at the lightest value of the passive quark mass, is taken as the central value. 
Table 6

Results of the linear fits to the Isgur-Wise function, at $\beta=6.0$. Quoted errors are statistical.

\begin{tabular}{lcccccc}
\hline & \multicolumn{2}{c}{$B \rightarrow D$} & \multicolumn{2}{c}{$B \rightarrow D^{*}$} & \multicolumn{2}{c}{ Sim. fit } \\
$\kappa_{\text {light }}$ & 0.13344 & 0.13417 & 0.13344 & 0.13417 & 0.13344 & 0.13417 \\
\hline$\rho^{2}$ & $0.94_{-16}^{+25}$ & $0.91_{-22}^{+32}$ & $0.68_{-34}^{+15}$ & $0.88_{-40}^{+25}$ & $0.88_{-18}^{+19}$ & $0.90_{-25}^{+25}$ \\
\hline
\end{tabular}

\section{Quark mass dependence}

Close to the heavy quark limit $\left(m_{b}, m_{c} \rightarrow \infty\right)$, at each fixed value of $\omega$, the Isgur-Wise function does not depend on the mass of the heavy quarks. However, the finite size of the simulated masses can introduce a quark mass dependence, which has to be examined.

\subsection{Light quark mass dependence}

In the dataset used in this work, only two values of the passive quark mass are available at each value of the coupling, making light quark mass extrapolations impossible. The values of the form factors with the two different light masses are compared and found to be statistically consistent with each other. However, the global fits of the Isgur-Wise function with the two different passive quarks yield slightly different, if still statistically compatible results. This effect is taken into account as a systematic uncertainty on the extracted slope of the Isgur-Wise function.

Figures 5 and 6 show a comparison of the fits of the Isgur-Wise function with the two different values of the light quark mass, at $\beta=6.2$, for both decays.

\subsection{Heavy quark mass dependence}

In this work the radiative corrections are applied, and the Isgur-Wise function is evaluated in a range in $\omega$ in which the lattice estimates of the power corrections for $h_{+}$and $h_{A_{1}}$ are found to be statistically consistent with zero. The Isgur-Wise function is extracted from the form factors by dividing off the radiative corrections. This procedure should remove all residual quark mass dependence at fixed $\omega$, if we are sufficiently close to the heavy quark limit. To study any residual heavy quark mass dependence, the Isgur-Wise function is extracted from all the momentum channels, holding all the quark masses fixed. For each heavy quark mass combination, the Isgur-Wise function is fitted, using all the kinematic channels, and its slope is extracted. Then, for each value 


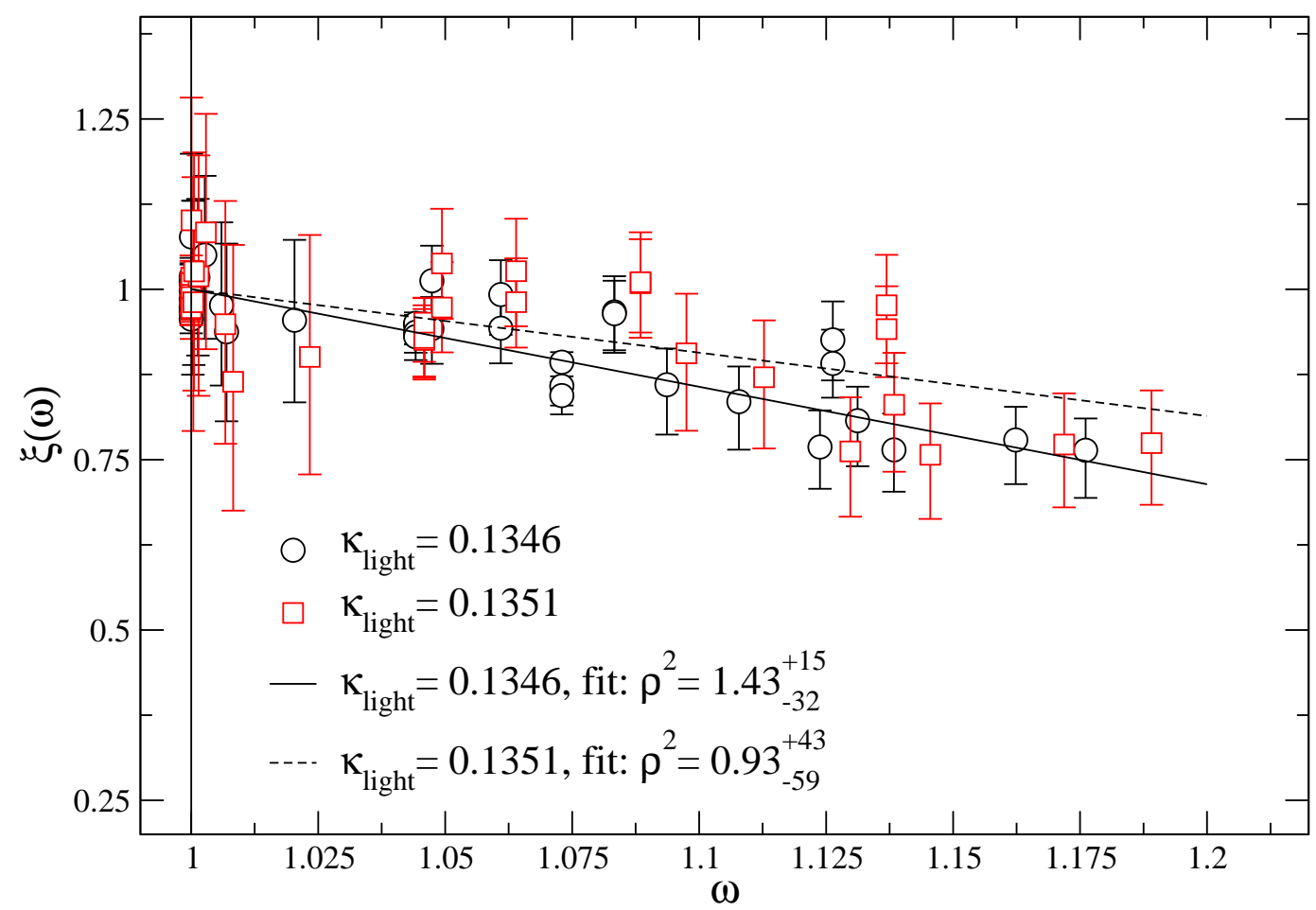

Fig. 5. The Isgur-Wise function from the $h_{A_{1}}$ form factor, for both values of the light quark mass, at $\beta=6.2$.

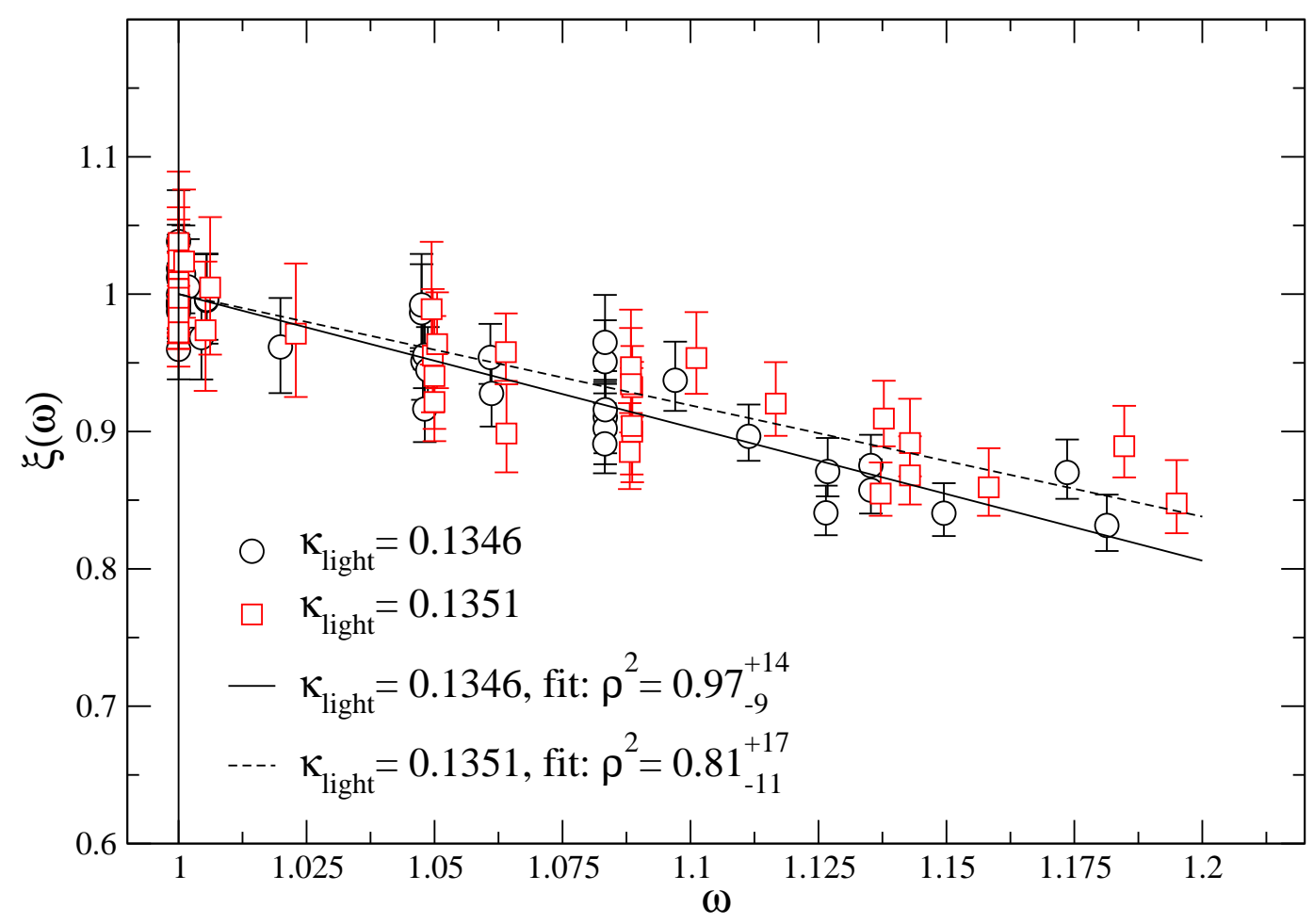

Fig. 6. The Isgur-Wise function from the $h_{+}$form factor, for both values of the light quark mass, at $\beta=6.2$. 


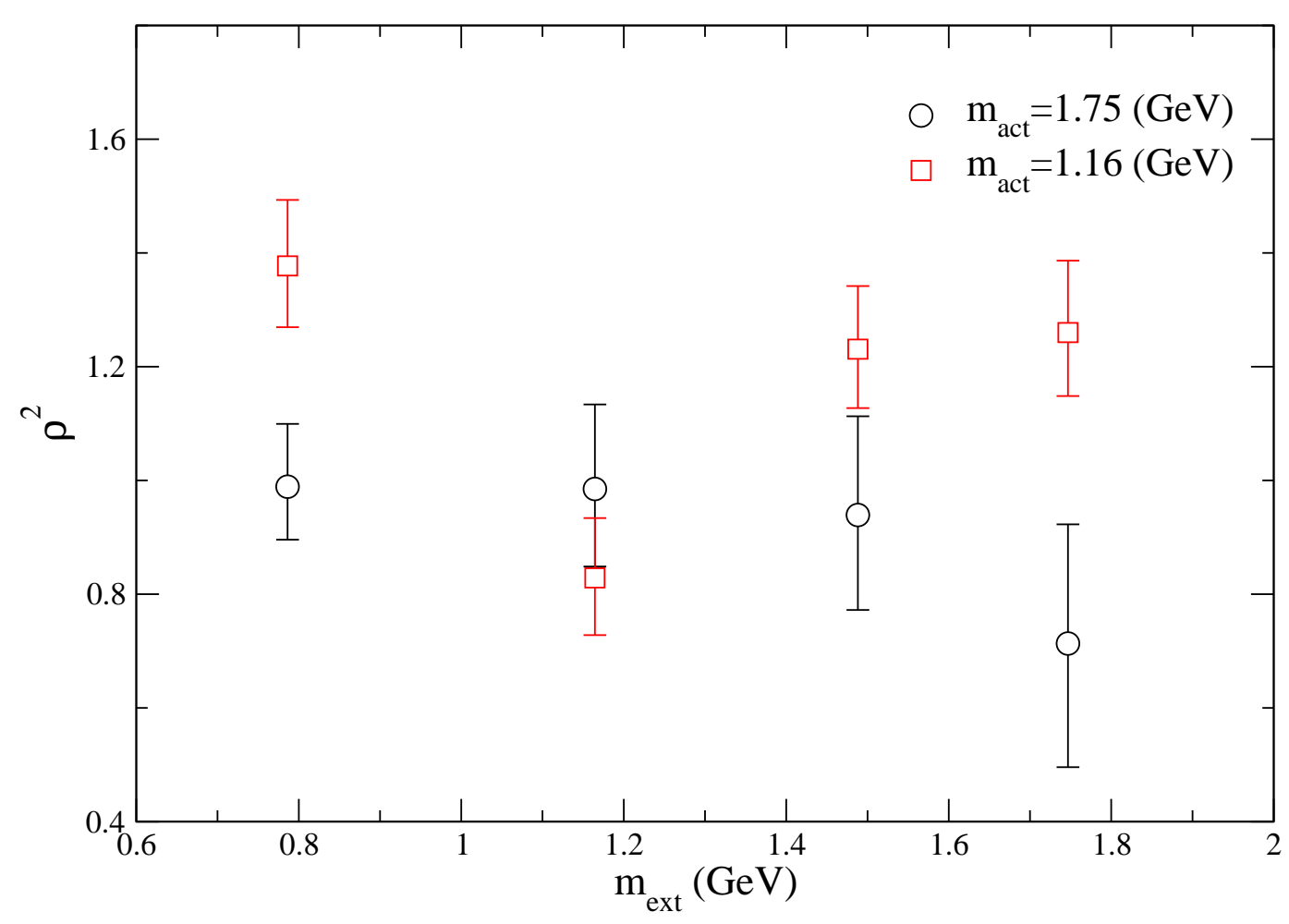

Fig. 7. Fits of the Isgur-Wise function at fixed values of the active quark propagator mass against the mass of the extended quark propagator, with $\kappa_{\text {light }}=0.1346$, at $\beta=6.2$. Quoted quark masses are improved and renormalised according to (13).

of the extended quark mass, the result of the fit is plotted against the value of the active quark mass. The results, for both values of $\beta$, at fixed values of the light quark mass, are shown in Figures 7 and 8. As one can see, there is no statistically significant heavy quark mass dependence. It should be noted that fixing the quark masses considerably reduces the number of points that are available to each of the fits: consequently, some of the fits are quite poor.

\section{Ratios of form factors}

The two following ratios of form factors are also calculated:

$$
\begin{aligned}
& R_{1}(\omega)=\frac{h_{V}(\omega)}{h_{A_{1}}(\omega)}, \\
& R_{2}(\omega)=\frac{h_{A_{3}}(\omega)+\frac{m_{D^{*}}}{m_{B}} h_{A_{2}}(\omega)}{h_{A_{1}}(\omega)} .
\end{aligned}
$$

These two ratios would be equal to one in the absence of symmetry breaking corrections. Figures 9 and 10 show that this work's determinations are in 


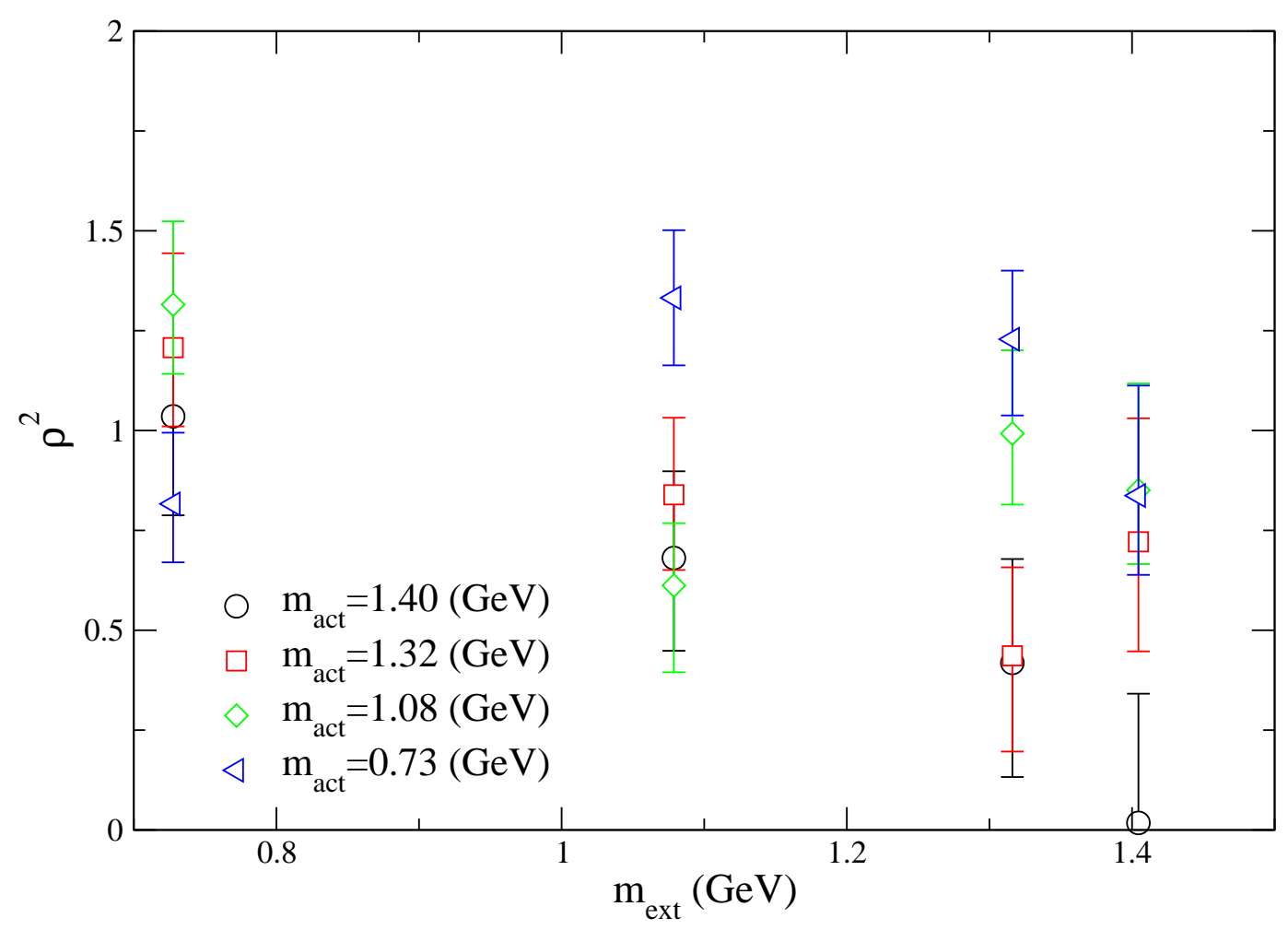

Fig. 8. Fits of the Isgur-Wise function at fixed values of the active quark propagator mass against the mass of the extended quark propagator, with $\kappa_{\text {light }}=0.13344$, at $\beta=6.0$. Quoted quark masses are improved and renormalised according to (13).

agreement with the experimental determinations by the CLEO collaboration [34], that quote

$$
\begin{aligned}
& R_{1}=1.18(32) \\
& R_{2}=0.71(23) .
\end{aligned}
$$

In the case of $R_{1}$ there is some evidence of a systematic deviation between our results and experiment. However, it should be noted that this form factor has a strong dependence on the improvement coefficient of the vector current, $c_{V}$, which is poorly known even at $\beta=6.2$.

\section{Systematic uncertainties and comparison with other results}

\subsection{Systematic uncertainties}

Several sources of systematic uncertainty are considered. Discretisation effects are estimated by comparing the results at the two values of $\beta$. The results at 


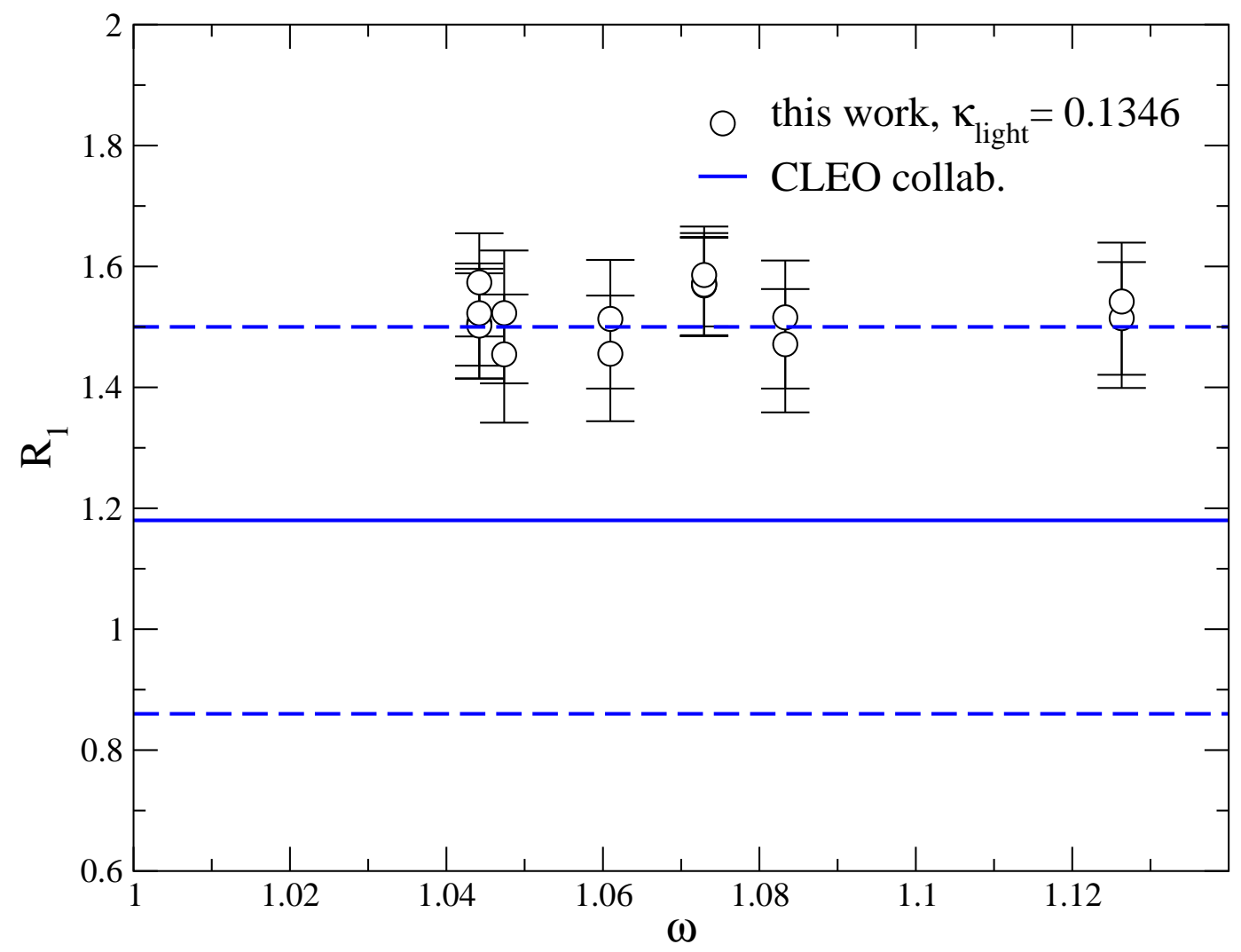

Fig. 9. The ratio $R_{1}$. The range of the experimental determination is shown by the dashed lines.

$\beta=6.2$ are taken as our central values. At $\beta=6.0$, the extraction of the form factors is affected by considerably larger statistical errors. This is reflected in the large statistical errors on the slope of the Isgur-Wise function.

The systematic uncertainty induced by the scale fixing quantity is evaluated by comparing fits of the Isgur-Wise function using $r_{0}$ and $m_{\rho}$ to set the scale. In all cases, it is found to be an effect smaller than $1 \%$. This is mainly due to the fact that the radiative corrections depend only logarithmically on the lattice spacing (through quark masses). Furthermore, the effect of the definition of the RGI quark mass and the values of the coefficients $Z_{m}$ and $b_{m}$ only enters through the radiative corrections.

The quark masses used in this calculation are around the charm quark mass. It is necessary to check for any residual heavy quark mass dependence beyond radiative and leading order power corrections. This has been examined and no statistically significant trend has been observed (see section 5.2). No numerical estimate for this is included in our systematic error. The light quark masses are around the strange quark mass, and thus the Isgur-Wise function extracted is $\xi_{s}$ rather than $\xi$. We examine the light quark mass dependence by comparing $\xi$ measured at the two values of quark mass. An extrapolation is not attempted, but the lightest mass is taken as a central value and the 


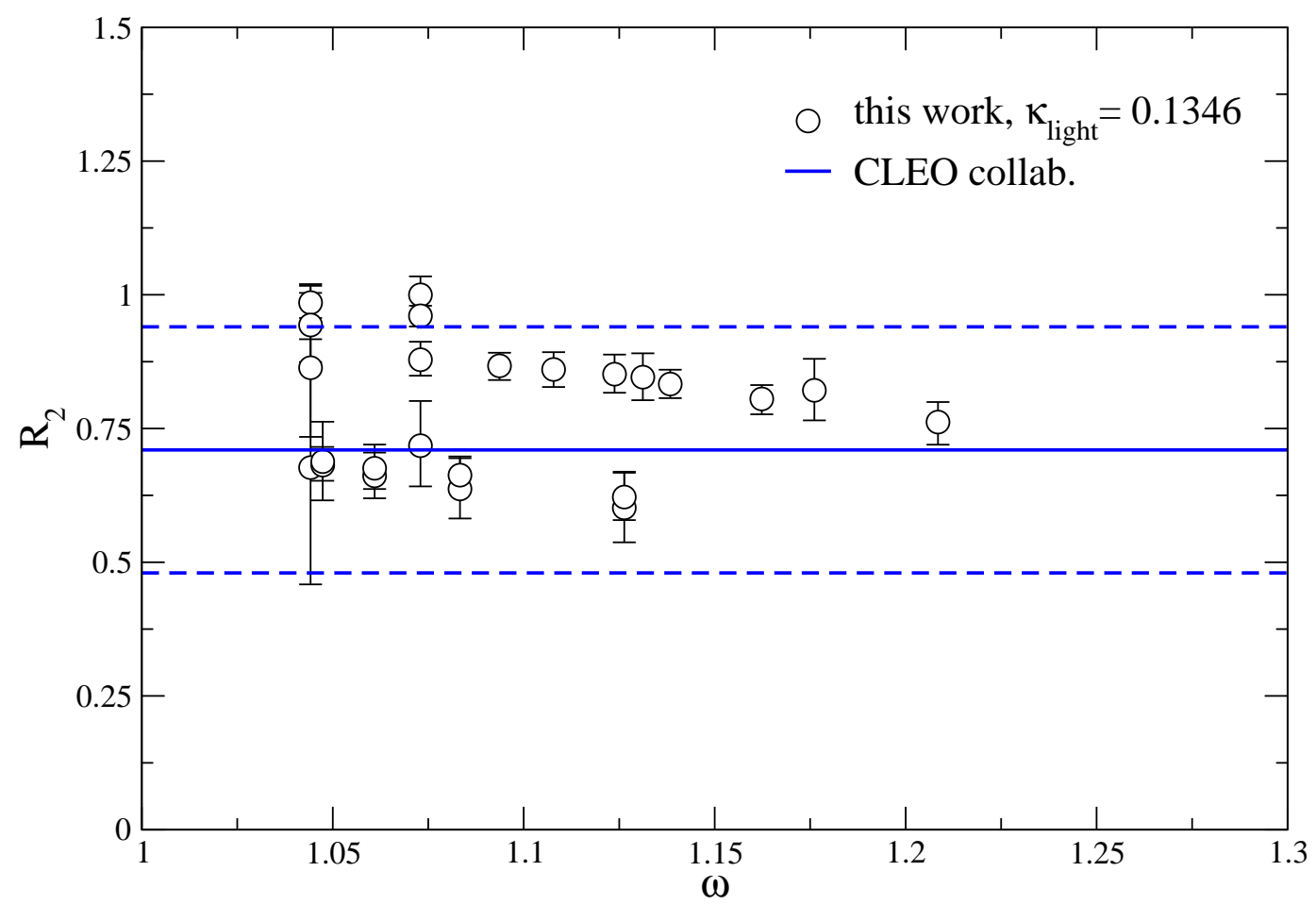

Fig. 10. The ratio $R_{2}$. The range of the experimental determination is shown by the dashed lines. The data points are scattered in a wide range because they correspond to different kinematic channels, with different systematic effects.

difference as a systematic uncertainty.

The statistical errors on $h_{A_{1}}$ are much larger than those on $h_{+}$and so the latter form factor dominates the simultaneous fit. Fitting to either form factor on its own produces the same result within statistical error. In all cases, the data satisfy the HQS condition $\xi(1)=1$ to such an extent that imposing this condition on the fit has no sizeable effect.

It is rather difficult to quantify the effects of quenching other than by performing the calculation in full QCD. There is some evidence that quenching could have a sizeable effect in heavy-light decay constants [35], around $10-15 \%$. However, the latter are dimensionful quantities and the scale setting ambiguity in the quenched approximation is sizeable. It is possible that large unquenching effects in the decay constant are at least partly due to the scale setting ambiguity [36]. The Isgur-Wise function is a dimensionless quantity and we have seen that it is almost independent of the choice of the scale fixing quantity. There is some evidence that quenching effects on the ratio $\xi_{s} / \xi_{d}$ are mild [37]. It is reasonable to assume that quenching effects will not be larger than any other systematic effect in this calculation.

Our systematic uncertainties are then the difference of $\xi$ obtained with the two values of the light quark mass $(+23 \%)$, with the two values of the cou- 
pling $(+8 \%)$ and with the quantity used to set the scale $(-1 \%)$. Positive and negative systematic uncertainties have been separately added in quadrature.

Therefore, our final result is

$$
\rho^{2}=0.83_{-11-1}^{+15+24}
$$

\subsection{Comparison with other results}

Other recent lattice results for heavy-light to heavy-light decays have concentrated on computing the value of the physical form factor at zero recoil to extract $\left|V_{c b}\right|$. They use ratios of matrix elements to cancel uncertainties [38] and further calculate the HQS breaking corrections [39]. Preliminary UKQCD results on this dataset for $h_{+}[40]$ and $h_{A_{1}}$ [41] are consistent with each other and this work. Previous UKQCD determinations [42-44] give $\rho^{2}=1.2_{-2-1}^{+2+2}$ with the spectator quark mass around strange, and $\rho^{2}=0.9_{-3-2}^{+2+4}$ when extrapolated to the chiral limit. Hashimoto and Matsufuru [45] compute $\xi$ using lattice HQET, but with an incomplete set of the $1 / m_{Q}$ corrections, and quote $\rho^{2}=0.70(17)$.

We also compare our result for the slope of the Isgur-Wise function to experiment. Both CLEO and Belle have recent measurements of $B \rightarrow D^{\star}$ decays $[46,47]$ and quote

$$
\begin{aligned}
& \rho^{2}=1.67(11)(22) \text { CLEO } \\
& \rho^{2}=1.35(17)(19) \text { Belle }
\end{aligned}
$$

Our result is significantly lower than the experimental ones, but consistent with other quenched lattice determinations. However, the experiments measure the slope of $\mathcal{F}(\omega)$, the physical form factor, which is equal to the IsgurWise function only in the infinite quark mass limit. Applying radiative corrections $\eta_{A}$ to our results would increase the slope by around $5 \%$. Considering the size of our uncertainties, this effect is small and we neglect it for this comparison.

\section{Acknowledgements}

The authors acknowledge PPARC grants PPA/G/O/1998/00621

and PPA/G/0/2000/00456. RDK acknowledges grant PPA/N/S/2000/00217, and CMM PPA/P/S/1998/00255. The authors thank J.M. Flynn and C. Mc- 
Neile for discussions. This work was supported by the European Community's Human potential programme under HPRN-CT-2000-00145 Hadrons/LatticeQCD

\section{References}

[1] S. Nussinov and W. Wetzel, Phys. Rev. D 36, 130 (1987).

[2] M. B. Voloshin and M. A. Shifman, Sov. J. Nucl. Phys. 45, 292 (1987).

[3] M. B. Voloshin and M. A. Shifman, Sov. J. Nucl. Phys. 47, 511 (1988).

[4] N. Isgur and M. B. Wise, Phys. Lett. B 232, 113 (1989).

[5] N. Isgur and M. B. Wise, Phys. Lett. B 237, 527 (1990).

[6] H. Georgi, Phys. Lett. B 240, 447 (1990).

[7] M. Neubert, Phys. Rev. D 46, 3914 (1992).

[8] M. Neubert, Phys. Rev. D 46, 2212 (1992).

[9] M. Luke, Phys. Lett. B 252, 447 (1990).

[10] N. Isgur, D. Scora, B. Grinstein, and M. Wise, Phys. Rev. D 39, 799 (1989).

[11] M. Neubert and V. Rieckert, Nucl. Phys. B 382, 97 (1992).

[12] B. Sheikholeslami and R. Wohlert, Nucl. Phys. B 259, 572 (1985).

[13] UKQCD Collaboration, K.C. Bowler et al., Nucl. Phys. B 619, 507 (2001).

[14] M. Lüscher, S. Sint, R. Sommer and P. Weisz, Nucl. Phys. B 478, 365 (1996).

[15] M. Lüscher, S. Sint, R. Sommer, P. Weisz, H. Wittig and U. Wolff, Nucl. Phys. B (Proc. Suppl.) 53, 905 (1997).

[16] T. Bhattacharya, R. Gupta, W. Lee, S. Sharpe, Nucl. Phys. B (Proc. Suppl.) 83-84, 851 (2000).

[17] T. Bhattacharya et al., Phys. Lett. B 461, 79 (1999).

[18] T. Bhattacharya, R. Gupta, W. Lee, S. Sharpe, Phys. Rev. D 62, 074505 (2001).

[19] S. Sint and P. Weisz, Nucl. Phys. B 502, 251 (1997).

[20] S. Capitani, M. Lüscher, R. Sommer, H. Wittig, Nucl. Phys. B 544, 669 (1999).

[21] R. Sommer, Nucl. Phys. B 411, 839 (1994).

[22] M. Guagnelli, R. Sommer and H. Wittig, Nucl. Phys. B 535, 389 (1998).

[23] M. Creutz, Phys. Rev. D 36, 2394 (1987).

[24] F. Brown and T. Woch, Phys. Rev. Lett. 58, 163 (1987). 
[25] N. Cabibbo and E. Marinari, Phys. Lett. B 119, 387 (1982).

[26] P. Lacock, A. McKerrell, C. Michael, I.M. Stopher and P.W. Stephenson, Phys. Rev. D 51, 6403 (1995).

[27] P. Boyle, hep-lat/9903033.

[28] B. Efron, SIAM Review 21, 460 (1979).

[29] C. Bernard and A. Soni, Nucl. Phys. B (Proc. Suppl.) 9, 155 (1989).

[30] C. Bernard, in Gauge Theory on a Lattice: 1984, Argonne National Laboratory Workshop, edited by C. Zachos et al. (National Technical Infomation Service, Springfield, VA, 1984).

[31] UKQCD Collaboration, K.C. Bowler et al., Phys. Rev. D 62, 054506 (2000).

[32] UKQCD Collaboration, K.C. Bowler et al., Phys. Lett. B 486, 111 (2000).

[33] M. Lüscher, S. Sint, R. Sommer, and H. Wittig, Nucl. Phys. B 491, 344 (1997).

[34] J.E. Duboscq et al., Phys. Rev. Lett. 76, 3898 (1996).

[35] S. Ryan, Nucl. Phys. B (Proc. Suppl.) 106, 86 (2002).

[36] UKQCD Collaboration, C.M. Maynard, Nucl. Phys. B (Proc. Suppl.) 106, 388 (2002).

[37] M.J. Booth, Phys. Rev. D 51, 2338 (1995).

[38] S. Hashimoto et al., Phys. Rev. D 61, 014502 (2000).

[39] S. Hashimoto et al., hep-ph/0110253.

[40] UKQCD collaboration, G. Douglas, Nucl. Phys. B (Proc. Suppl.) 83, 280 (2000).

[41] UKQCD collaboration, G.N. Lacagnina, Nucl. Phys. B (Proc. Suppl.) 106, 373 (2002).

[42] UKQCD Collaboration, S. P. Booth, Phys. Rev. Lett. 72, 462 (1994).

[43] UKQCD Collaboration, L. Lellouch, Phys. Proc. Supp. 42, 421 (1995).

[44] UKQCD Collaboration, K. C. Bowler, Phys. Rev. D 52, 5067 (1995).

[45] S. Hashimoto and H. Matsufuru, Phys. Rev. D 54, 4578 (1996).

[46] CLEO collaboration, J.P. Alexander et al., hep-ex/0007052.

[47] BELLE collaboration, K. Abe et al., hep-ex/0111060. 